\title{
A SIMPLE ALGEBRAIC PROOF OF THE ALGEBRAIC INDEX THEOREM
}

\author{
Poning Chen And Vasiliy Dolgushev
}

\begin{abstract}
In math.QA/0311303 B. Feigin, G. Felder, and B. Shoikhet proposed an explicit formula for the trace density map from the quantum algebra of functions on an arbitrary symplectic manifold $\mathcal{M}$ to the top degree cohomology of $\mathcal{M}$. They also evaluated this map on the trivial element of $K$-theory of the algebra of quantum functions. In our paper we evaluate the map on an arbitrary element of $K$-theory, and show that the result is expressed in terms of the $\hat{A}$-genus of $\mathcal{M}$, the Deligne-Fedosov class of the quantum algebra, and the Chern character of the principal symbol of the element. For a smooth (real) symplectic manifold (without a boundary), this result implies the Fedosov-Nest-Tsygan algebraic index theorem.
\end{abstract}

\section{Introduction}

The famous Atiyah-Singer index theorem [2] relates the index of an elliptic pseudo-differential operator on a compact manifold $X$ to the Todd class of $X$ and the Chern character of the bundle naturally associated with the symbol of the pseudo-differential operator. It is well-known that the algebra of pseudodifferential operators can be viewed as a "quantization" of the cotangent bundle $T^{*} X$. Starting from this point B. Fedosov [9] proposed a natural analogue of the Atiyah-Singer index theorem for the deformation quantization of an arbitrary symplectic manifold. This theorem expresses the index of a compactly supported element in $K$-theory of the quantum algebra of functions on a symplectic manifold $\mathcal{M}$ via the $\hat{A}$-genus of $\mathcal{M}$, the Deligne-Fedosov class of the quantum algebra, and the Chern character of the principal symbol of the $K$-theory element. While the original index theorem of Atiyah and Singer relates two integers, the Fedosov index theorem relates two formal power series with complex coefficients.

In paper [16] R. Nest and B. Tsygan proposed a completely different proof of the algebraic index theorem based on the relations between Hochschild (co)homology, cyclic (co)homology, and Lie algebra (co)homology. In their next paper [18] they also showed that the analytic index theorem for a compact manifold (without a boundary) could be derived from the algebraic one. In order to generalize this result to manifolds with a boundary (or more generally, with corners) one is in great need of a local version of the algebraic index theorem which relates De Rham cohomology classes, rather than formal power series of complex numbers.

Received by the editors November 21, 2004. 
The aim of this paper is to give such a version (see theorem 4 in section 3) using the trace density map proposed in paper [10] by B. Feigin, G. Felder, and B. Shoikhet. Namely, we show that the Feigin-Felder-Shoikhet (FFS) trace density map sends an arbitrary element of $K$-theory of the algebra of quantum functions on $\mathcal{M}$ to the top component of the cup product of the $\hat{A}$-genus of $\mathcal{M}$, the exponent of the Deligne-Fedosov class of the quantum algebra, and the Chern character of the principal symbol of the element. Thus, we generalize the result of [10], in which the trace density map was evaluated at the trivial element of $K$-theory. For the case of the cotangent bundle this computation of [10] was performed in [7].

We have to mention that in [6] another version of the trace density map was proposed. This map sends a cohomology class of the sheaf of the periodic cyclic complex $C C_{\bullet}^{\text {per }}\left(\mathcal{O}_{X}^{\text {quant }}\right)$ of the structure sheaf $\mathcal{O}_{X}^{\text {quant }}$ of quantum functions on a holomorphic symplectic manifold $X$ to the cohomology of the constant sheaf on $X$. In [6] it was proven that the evaluation of this map on an arbitrary cohomology class of the sheaf of periodic cyclic complex $C C_{\bullet}^{\text {per }}\left(\mathcal{O}_{X}^{\text {quant }}\right)$ can be expressed in terms of the principal symbol of the class, $\hat{A}$-genus of $X$ and the Deligne-Fedosov class of the quantum deformation. In [6] the authors refer to this result as a Riemann-Roch theorem for deformation quantizations. It is not hard to see that the local version of the algebraic index theorem proven in our paper follows from the results of P. Bressler, R. Nest and B. Tsygan [6]. However, our proof is more explicit and straightforward. For this reason we expect that our technique is more powerful for exploring the parallel analytic results.

We would like to mention an alternative deformation quantization procedure due to L. Boutet de Monvel and V. Guillemin [4]. This procedure allows us to quantize an arbitrary compact symplectic manifold with an integral symplectic form. Recently R. Melrose proposed in [15] a beautiful idea on how one can relax the integrality condition in the quantization of L. Boutet de Monvel and V. Guillemin. R. Melrose suggests that using this procedure one could derive the algebraic index theorem from the analytic one.

The organization of the paper is as follows. In section two, we give a reminder of Fedosov's construction [9] of the deformation quantization with twisted coefficients on a symplectic manifold. In this section we also recall the necessary results of Feigin, Felder, and Shoikhet about their trace density map proposed in [10]. In section three, we prove the local version of the algebraic index theorem (see theorem 4), using the FFS trace density map. In the concluding section we make a few remarks about the local algebraic index theorem for symplectic Lie algebroids and the versions of the local Riemann-Roch-Hirzebruch theorem.

Throughout the paper we assume the summation over repeated indices. We assume that $\mathcal{M}$ is either a smooth real symplectic manifold of dimension $2 n$ or a smooth affine algebraic variety (over $\mathbb{C}$ ) of the complex dimension $2 n$, endowed with an algebraic symplectic form. $C(\mathcal{M})$ stands, respectively, for the algebra of smooth function or for the algebra of regular functions on $\mathcal{M}$. The notation $S_{k}$ is reserved for the group of permutations of $k$ elements. We omit 
the symbol $\wedge$ referring to a local basis of exterior forms, as if we thought of $d x^{i}$ 's as anti-commuting variables. For a vector space $\mathfrak{h}$ we denote by $S^{j} \mathfrak{h}$ the subspace of monomials of degree $j$ in the symmetric algebra $S \mathfrak{h}$ of $\mathfrak{h}$. Finally, we always assume that a nilpotent linear operator is the one whose second power is vanishing.

\section{Preliminaries}

In this section we review the Fedosov deformation quantization of endomorphisms of a vector bundle $V$ over a symplectic manifold $\mathcal{M}$ (See [9], section 5.3). We also recall the Felder-Feigin-Shoikhet construction [10] of the trace density map.

2.1. The Feigin-Felder-Shoikhet cocycles. First, we recall that for any associative algebra $\mathcal{A}$ with a unit over a field $K$ (of characteristic zero) we have a chain map $\phi^{N}$ from the Hochschild cochain complex $C^{\bullet}\left(\mathcal{A}, \mathcal{A}^{*}\right)$ with coefficients in the dual module $\mathcal{A}^{*}$ to the Lie algebra cochain complex $C^{\bullet}\left(\mathfrak{g l}_{N}(\mathcal{A}) ; \mathfrak{g l}_{N}(\mathcal{A})^{*}\right)$ with coefficients in $\mathfrak{g l}_{N}(\mathcal{A})^{*}$

$$
\begin{gathered}
\phi^{N}(\psi)\left(M_{1} \otimes a_{1}, \ldots, M_{k} \otimes a_{k}\right)\left(M_{0} \otimes a_{0}\right) \\
=\frac{1}{k !} \sum_{\nu \in S_{k}}(-)^{\nu} \psi\left(a_{\nu(1)} \otimes \cdots \otimes a_{\nu(k)}\right)\left(a_{0}\right) \operatorname{tr}\left(M_{0} M_{\nu(1)} \ldots M_{\nu(k)}\right),
\end{gathered}
$$

where $M_{l} \in \mathfrak{g l}_{N}(K), a_{l} \in \mathcal{A}$, and $\psi \in C^{k}\left(\mathcal{A}, \mathcal{A}^{*}\right)$.

Let $\mathcal{V}$ be a $2 n$-dimensional vector space (over $\mathbb{C}$ ) endowed with a symplectic form $B$. Let $\left\{y^{1}, \ldots, y^{2 n}\right\}$ be a basis in $\mathcal{V}$. We denote by $\left\|B^{i j}\right\|,(i, j=1, \ldots, 2 n)$ the matrix

$$
B^{i j}=B\left(y^{i}, y^{j}\right)
$$

of the form $B$ in this basis. Let $\varepsilon$ be the completely antisymmetric Liouville tensor whose components $\varepsilon^{i_{1} \ldots i_{2 n}}$ are defined in the basis $\left\{y^{1}, \ldots, y^{2 n}\right\}$ as follows

$$
\varepsilon^{i_{1} \ldots i_{2 n}}=\frac{(-1)^{n}}{n !} \sum_{\nu \in S_{2 n}}(-)^{\nu} B^{i_{\nu(1)} i_{\nu(2)}} \ldots B^{i_{\nu(2 n-1)} i_{\nu(2 n)}} .
$$

Definition 1. The Weyl algebra $\mathcal{W}$ associated with the symplectic vector space $\mathcal{V}$ is the vector space $\mathbb{C}[[\mathcal{V}]]((\hbar))$ of the formally completed symmetric algebra of $\mathcal{V}$ equipped with the following (associative) multiplication

$$
(a \circ b)(y, \hbar)=\left.\exp \left(\frac{\hbar}{2} B^{i j} \frac{\partial}{\partial y^{i}} \frac{\partial}{\partial z^{j}}\right) a(y, \hbar) b(z, \hbar)\right|_{y=z} .
$$

One can easily see that the multiplication defined by (3) does not depend on the choice of a basis in $\mathcal{V}$. We view $\mathcal{W}$ as an algebra over the field $\mathbb{C}((\hbar))$. 
The Weyl algebra $\mathcal{W}$ is naturally filtered with respect to the degree of monomials $2[\hbar]+[y]$ where $[\hbar]$ is a degree in $\hbar$ and $[y]$ is a degree in $y$

$$
\begin{gathered}
\cdots \subset \mathcal{W}^{1} \subset \mathcal{W}^{0} \subset \mathcal{W}^{-1} \ldots \subset \mathcal{W}, \\
\mathcal{W}^{m}=\left\{a=\sum_{2 k+p \geq m} \hbar^{k} a_{k ; i_{1} \ldots i_{p}} y^{i_{1}} \ldots y^{i_{p}}\right\}
\end{gathered}
$$

This filtration defines the $2[\hbar]+[y]$-adic topology in $\mathcal{W}$.

In [10] Feigin, Felder, and Shoikhet proposed an explicit expression for the $(2 n)$ th Hochschild (continuous) cocycle of the Weyl algebra $\mathcal{W}$ with coefficients in the dual module $\mathcal{W}^{*}$. Their formula is reminiscent of Kontsevich's construction of the structure maps of his celebrated formality quasi-isomorphism [13]. However, unlike the integrals in Kontsevich's construction [13] the coefficients entering the formula of Feigin, Felder, and Shoikhet are rational.

Following [10] we start with the $2 n$-th simplex

$$
\Delta_{2 n}=\left\{\left(u_{1}, \ldots, u_{2 n}\right) \in \mathbb{R}^{2 n} \mid 0 \leq u_{1} \cdots \leq u_{2 n} \leq 1\right\}
$$

with the standard orientation and denote by $B_{b c}$ the action of the form $B$ on the $b$-th and $c$-th components of $\mathcal{W}^{\otimes(2 n+1)}$

$$
\begin{gathered}
B_{b c}\left(a_{0} \otimes \cdots \otimes a_{2 n}\right)= \\
B^{i j}\left(a_{0} \otimes \cdots \otimes \frac{\partial a_{b}}{\partial y^{i}} \otimes \cdots \otimes \frac{\partial a_{c}}{\partial y^{j}} \otimes \cdots \otimes a_{2 n}\right), \quad a_{b} \in \mathcal{W} .
\end{gathered}
$$

Furthermore, we denote by $\pi_{2 n}$ the action of the Liouville tensor (2)

$$
\pi_{2 n}\left(a_{0} \otimes \cdots \otimes a_{2 n}\right)=\varepsilon^{i_{1} \ldots i_{2 n}} a_{0} \otimes \frac{\partial a_{1}}{\partial y^{i_{1}}} \otimes \cdots \otimes \frac{\partial a_{2 n}}{\partial y^{i_{2 n}}} .
$$

Finally, if we denote by $\mu$ the natural projection from $\left(\mathcal{W}^{\otimes(2 n+1)}\right)$ onto $\mathbb{C}((\hbar))$

$$
\mu\left(a_{0} \otimes \cdots \otimes a_{2 n}\right)=a_{0}(0) \ldots a_{2 n}(0),
$$

then the Feigin-Felder-Shoikhet formula of the $2 n$-th Hochschild cocycle $\tau_{2 n} \in C^{2 n}\left(\mathcal{W},(\mathcal{W})^{*}\right)$ can be written as $[10]$

$\tau_{2 n}(\alpha)\left(a_{0}\right)=\mu_{2 n}\left(\int_{\Delta_{2 n}} \prod_{0 \leq b \leq c \leq 2 n} e^{\frac{\hbar}{2}\left(2 u_{b}-2 u_{c}+1\right) B_{b c}} \pi_{2 n}\left(a_{0} \otimes \alpha\right) d u_{1} \wedge \cdots \wedge d u_{2 n}\right)$,

where $a_{0} \in \mathcal{W}, \alpha \in \mathcal{W}^{\otimes(2 n)}$, and $u_{0}=0$. It is easy to see that the cocycle $\tau_{2 n}$ does not depend on the choice of the basis $\left\{y^{1}, \ldots, y^{2 n}\right\}$ in $\mathcal{V}$.

Applying the map (1) to (5) we get the $2 n$-th cocycle in the chain complex $C^{\bullet}\left(\mathfrak{g l}_{N}(\mathcal{W}), \mathfrak{g l}_{N}(\mathcal{W})^{*}\right)$ of the Lie algebra $\mathfrak{g l}_{N}(\mathcal{W})$ with values in the dual module $\left(\mathfrak{g l}_{N}(\mathcal{W})\right)^{*}$

$$
\Theta_{2 n}^{N}=\phi^{N}\left(\tau_{2 n}\right): \wedge^{2 n}\left(\mathfrak{g l}_{N}(\mathcal{W})\right) \otimes \mathfrak{g l}_{N}(\mathcal{W}) \mapsto \mathbb{C}((\hbar)) .
$$

In [10] it was shown that the latter cocycle satisfies remarkable properties which allow us to construct a trace density map for a quantum algebra of functions on any symplectic manifold. Before talking about these properties we recall a necessary construction of the Chern-Weil theory. 
2.2. The Chern-Weil homomorphism. Let $\mathfrak{g}$ be a Lie algebra and $\mathfrak{h} \subset \mathfrak{g}$ be a subalgebra of $\mathfrak{g}$. Suppose that there is an $\mathfrak{h}$-equivariant projection $p r: \mathfrak{g} \mapsto \mathfrak{h}$, that is a map commuting with the adjoint action of $\mathfrak{h}$ and satisfying the property $\left.p r\right|_{\mathfrak{h}}=I d_{\mathfrak{h}}$. The amount by which $p r$ fails to be a Lie algebra homomorphism is measured by the "curvature" $C \in \operatorname{Hom}\left(\wedge^{2} \mathfrak{g}, \mathfrak{h}\right)$

$$
C(v, w)=[p r(v), \operatorname{pr}(w)]-\operatorname{pr}([v, w]), \quad v, w \in \mathfrak{g} .
$$

Given the "curvature" (7) of the $\mathfrak{h}$-equivariant projection $p r$, it is not hard to see that for any adjoint invariant form $Q \in\left(\left(S^{j} \mathfrak{h}\right)^{*}\right)^{\mathfrak{h}}$ the formula (8)

$$
\chi(Q)\left(v_{1}, \ldots, v_{2 j}\right)=\frac{1}{(2 j) !} \sum_{\nu \in S_{2 j}}(-)^{\nu} Q\left(C\left(v_{\nu(1)}, v_{\nu(2)}\right), \ldots, C\left(v_{\nu(2 j-1)}, v_{\nu(2 j)}\right)\right)
$$

defines a relative Lie algebra cocycle $\chi(Q) \in C^{2 j}(\mathfrak{g}, \mathfrak{h})$. The standard argument of the theory of characteristic classes shows that the cohomology class $[\chi(Q)]$ of the cocycle $\chi(Q)$ does not depend on the choice of the projection $p r$. Thus (8) induces a map from the vector space $\left(\left(S^{j} \mathfrak{h}\right)^{*}\right)^{\mathfrak{h}}$ to $H^{2 j}(\mathfrak{g}, \mathfrak{h})$. The latter map is called the Chern-Weil homomorphism.

In our case we set $\mathfrak{g}=\mathfrak{g l}_{N}(\mathcal{W}), \mathfrak{h}=\mathfrak{g l}_{N} \oplus \mathfrak{s p}_{2 n}$ and define the projection $p r$ from $\mathfrak{g}$ to $\mathfrak{h}$ by the formula

$$
\begin{gathered}
p r(v)=p r_{0}(v)+p r_{2}(v), \\
p r_{0}(v)=\left.v\right|_{y=0}, \quad p r_{2}(v)=\frac{1}{N} \sigma_{2}(\operatorname{tr}(v)) I_{N},
\end{gathered}
$$

where $\sigma_{2}$ denotes the projection onto the monomials of the second degree in $y$ 's, $t r$ is the ordinary matrix trace, $I_{N}$ is the identity matrix of size $N \times N$, and the Lie algebra $\mathfrak{s p}_{2 n}$ is realized as a subalgebra of scalar matrices in $\mathfrak{g l}_{N}(\mathcal{W})$ with values in quadratic monomials in $\mathcal{W}$.

Due to [10] we have the following

Theorem 1 (Feigin-Felder-Shoikhet [10]). The Lie algebra cocycle $\Theta_{2 n}^{N} \in C^{2 n}\left(\mathfrak{g}, \mathfrak{g}^{*}\right)$ is relative with respect to the subalgebra $\mathfrak{h}=\mathfrak{g l}_{N} \oplus \mathfrak{s p}_{2 n}$. The evaluation of (6) on the identity matrix $I_{N} \in \mathfrak{g}$ gives a relative Lie algebra cocycle

$$
\begin{gathered}
\varphi \in C^{2 n}(\mathfrak{g}, \mathfrak{h}) \\
\varphi=\Theta_{2 n}^{N}\left(\cdot, \ldots, \cdot, I_{N}\right): \wedge^{2 n}(\mathfrak{g}) \mapsto \mathbb{C}((\hbar))
\end{gathered}
$$

whose cohomology class

$$
[\varphi]=\left[\chi\left(Q_{n}\right)\right]
$$

coincides with the image of the $n$-th component $Q_{n} \in\left(\left(S^{n} \mathfrak{h}\right)^{*}\right)^{\mathfrak{h}}$ of the adjoint invariant form $Q \in\left((S \mathfrak{h})^{*}\right)^{\mathfrak{h}}$

$$
\begin{gathered}
Q(X, \ldots, X)=\operatorname{det}\left(\frac{X_{1} / 2 \hbar}{\sinh \left(X_{1} / 2 \hbar\right)}\right)^{1 / 2} \operatorname{tr} \exp \left(\frac{X_{2}}{\hbar}\right), \\
X=X_{1} \oplus X_{2} \in \mathfrak{s p}_{2 n} \oplus \mathfrak{g l}_{N}
\end{gathered}
$$

under the Chern-Weil homomorphism (8). 
2.3. Fedosov deformation quantization with twisted coefficients. Let $\mathcal{M}$ be a symplectic manifold of dimension $2 n$. We denote by $\omega=\omega_{i j}(x) d x^{i} d x^{j}$ the corresponding symplectic form and by

$$
\bar{\omega}=\omega^{i j}(x) \frac{\partial}{\partial x^{i}} \wedge \frac{\partial}{\partial x^{j}}
$$

the corresponding Poisson tensor. Here $x^{i}$ denote local coordinates and the indices $i, j$ run from 1 from $2 n$. For a vector bundle $V$ of rank $N$ over $\mathcal{M}$ we will denote by $\operatorname{End}(V)$ the bundle of endomorphisms of $V$ and by $E n d_{V}=$ $\Gamma(\mathcal{M}, \operatorname{End}(V))$ the algebra of global sections of $\operatorname{End}(V)$.

If the vector bundle $V$ is endowed with a connection $\partial^{V}$ then

Definition 2. By quantization of the algebra End $d_{V}$ we mean a construction of an associative $\mathbb{C}((h))$-linear product in $\operatorname{End}_{V}((\hbar))$ given by the formal power series

$$
a * b=a b+\sum_{k \geq 1} \hbar^{k} B_{k}(a, b), \quad a, b \in \operatorname{End}_{V}
$$

of bidifferential operators $B_{k}:$ End $_{V} \otimes$ End $_{V} \mapsto$ End $_{V}$ such that

$$
B_{1}(a, b)-B_{1}(b, a)=\omega^{i j}(x) \partial_{i}^{V}(a) \partial_{j}^{V}(b) .
$$

In [9] B. Fedosov proposed a simple procedure for the deformation quantization of the algebra of endomorphisms of a vector bundle $V$ over a symplectic manifold $\mathcal{M}$. The main ingredient of the construction is the Weyl algebra bundle $W\left(E n d_{V}\right)$ whose sections are the following formal power series

$$
a=a(x, y, h)=\sum_{k, l} \hbar^{k} a_{k ; i_{1} i_{2} \ldots i_{l}}(x) y^{i_{1}} \ldots y^{i_{l}},
$$

where $y=\left(y^{1} \ldots y^{2 n}\right)$ are fiber coordinates of the tangent bundle $T \mathcal{M}, a_{k ; i_{1} i_{2} \ldots i_{l}}$ represent sections of $\operatorname{End}(V) \otimes S^{l}\left(T^{*} M\right)$ and the summation over $k$ is bounded below.

Multiplication of two sections of $W\left(E n d_{V}\right)$ is given by the Weyl formula

$$
a \circ b(x, y, \hbar)=\left.\exp \left(\frac{\hbar}{2} \omega^{i j} \frac{\partial}{\partial y^{i}} \frac{\partial}{\partial z^{j}}\right) a(x, y, \hbar) b(x, z, \hbar)\right|_{y=z} .
$$

Notice that in the right hand side of (14) the sections of $W\left(E n d_{V}\right)$ are multiplied via the product induced from the algebra $E n d_{V}$.

For any point $p \in \mathcal{M}$ the fiber $W_{p}\left(E n d_{V}\right)$ of the Weyl algebra bundle at $p$ is isomorphic to the algebra $\mathfrak{g l}_{N}(\mathcal{W})$ of $N \times N$-matrices of the Weyl algebra $\mathcal{W}$ associated with the cotangent space $T_{p}^{*}(\mathcal{M})$ at the point $p$ with the symplectic form $\bar{\omega}_{p}$. The transition functions are realized by the adjoint action of the group $G L_{N}$. 
The filtration (4) of the Weyl algebra gives us a natural filtration of the bundle $W\left(\operatorname{End}_{V}\right)$

$$
\begin{gathered}
\cdots \subset W^{1}\left(E n d_{V}\right) \subset W^{0}\left(E n d_{V}\right) \subset W^{-1}\left(E n d_{V}\right) \cdots \subset W\left(E n d_{V}\right), \\
\Gamma\left(W^{m}\left(E n d_{V}\right)\right)=\left\{a=\sum_{2 k+p \geq m} \hbar^{k} a_{k ; i_{1} \ldots i_{p}}(x) y^{i_{1}} \ldots y^{i_{p}}\right\} .
\end{gathered}
$$

This filtration defines the $2[\hbar]+[y]$-adic topology in the algebra $\Gamma\left(W\left(E n d_{V}\right)\right)$ of sections of $W\left(E n d_{V}\right)$.

The vector space $\Omega^{\bullet}\left(W\left(E n d_{V}\right)\right)$ of smooth exterior forms with values in $W\left(E n d_{V}\right)$ is naturally a graded associative algebra with the product induced by (14) and the following graded commutator

$$
[a, b]=a \circ b-(-)^{q_{a} q_{b}} b \circ a,
$$

where $q_{a}$ and $q_{b}$ are exterior degrees of $a$ and $b$, respectively. The filtration of $W\left(E n d_{V}\right)(15)$ gives us a filtration of the algebra $\Omega^{\bullet}\left(W\left(E n d_{V}\right)\right)$

$$
\begin{aligned}
\cdots \subset \Omega^{\bullet} & \left(W^{1}\left(E n d_{V}\right)\right) \subset \Omega^{\bullet}\left(W^{0}\left(E n d_{V}\right)\right) \subset \\
& \subset \Omega^{\bullet}\left(W^{-1}\left(E n d_{V}\right)\right) \subset \cdots \subset \Omega^{\bullet}\left(W\left(E n d_{V}\right)\right) .
\end{aligned}
$$

In what follows we refer to the algebra $\Omega^{\bullet}(\mathcal{M})$ of exterior forms on $\mathcal{M}$ as an algebra embedded into $\Omega^{\bullet}\left(W\left(E n d_{V}\right)\right)$ via the natural map $\iota: \Omega^{\bullet}(\mathcal{M}) \mapsto$ $\Omega^{\bullet}\left(W\left(E n d_{V}\right)\right)$, which sends an exterior form $\eta \in \Omega^{\bullet}(\mathcal{M})$ to the scalar matrix $\eta I_{N} \in \Omega^{\bullet}\left(W\left(E n d_{V}\right)\right)$.

Let $\partial^{s}$ be a torsion free connection on $T \mathcal{M}$ compatible with the symplectic structure $\omega$. Using the connection $\partial^{s}$ and the connection $\partial^{V}$ on $V$ we define the following linear operator

$$
\begin{aligned}
& \nabla: \Omega^{\bullet}\left(W\left(\operatorname{End}_{V}\right)\right) \mapsto \Omega^{\bullet+1}\left(W\left(\operatorname{End}_{V}\right)\right), \\
& \nabla=d x^{i} \frac{\partial}{\partial x^{i}}-d x^{i} \Gamma_{i j}^{k}(x) y^{j} \frac{\partial}{\partial y^{k}}+\left[\Gamma_{V}, \cdot\right]
\end{aligned}
$$

where $\Gamma_{i j}^{k}(x)$ are Christoffel symbols of $\partial^{s}$ and $\Gamma^{V}$ is the connection form of $\partial^{V}$.

Thanks to the compatibility of $\partial^{s}$ with the symplectic structure $\omega$ the operator (16) is a derivation of the graded algebra $\Omega^{\bullet}\left(W\left(E n d_{V}\right)\right)$. Furthermore, a simple computation shows that

$$
\nabla^{2} a=\frac{1}{2}\left[R+R^{V}, a\right], \quad \forall a \in \Omega\left(W\left(E n d_{V}\right)\right),
$$

where $R^{V} \in \Omega^{2}\left(E n d_{V}\right)$ is the curvature form of $\partial^{V}$,

$$
R=\frac{1}{2 \hbar} \omega_{k m}\left(R_{i j}\right)_{l}^{m}(x) y^{k} y^{l} d x^{i} d x^{j},
$$

and $\left(R_{i j}\right)_{l}^{m}(x)$ is the Riemann curvature tensor of $\partial^{s}$. 
Definition 3. The Fedosov connection is a nilpotent derivation of the graded algebra $\Omega^{\bullet}\left(W\left(\right.\right.$ End $\left.\left._{V}\right)\right)$ of the following form

$$
D=\nabla+\frac{1}{\hbar}[A, \cdot], \quad A=-d x^{i} \omega_{i j}(x) y^{j}+r,
$$

where $r$ is an element in $\Omega^{1}\left(W^{2}\left(E n d_{V}\right)\right)$

The flatness of $D$ is equivalent to the fact that the Fedosov-Weyl curvature

$$
C^{W}=\hbar\left(R+R^{V}\right)+2 \nabla A+\frac{1}{\hbar}[A, A]
$$

of $D$ belongs to the subspace $\Omega^{2}(\mathcal{M})((\hbar)) \subset \Omega^{2}\left(W\left(E n d_{V}\right)\right)$. A simple analysis of degrees in $\hbar$ and $y$ shows that $C^{W}$ is of the form

$$
C^{W}=-\omega+\Omega_{\hbar}, \quad \Omega_{h} \in \hbar \Omega^{2}(M)[[\hbar]]
$$

whereas the Bianchi identity $D\left(C^{W}\right)=0$ implies that $\Omega_{h}$ is a series of two-forms closed with respect to the De Rham differential.

One can observe that the definition of the Fedosov connection depends on the choice of the symplectic connection $\partial^{s}$ and the connection $\partial^{V}$ on $V$. The following proposition shows how this problem can be remedied

Proposition 1. If $\nabla$ and $\widetilde{\nabla}$ are two operator (16) corresponding to the symplectic connections $\partial^{s}, \widetilde{\partial}^{s}$ and the connections $\partial^{V}, \widetilde{\partial}^{V}$ on $V$, respectively, then the difference $\widetilde{D}-D$ between two Fedosov connections

$$
\widetilde{D}=\widetilde{\nabla}+\frac{1}{\hbar}\left[-d x^{i} \omega_{i j}(x) y^{j}+\widetilde{r}, \cdot\right], \quad D=\nabla+\frac{1}{\hbar}\left[-d x^{i} \omega_{i j}(x) y^{j}+r, \cdot\right],
$$

takes the form of the commutator

$$
\widetilde{D}-D=\frac{1}{\hbar}[\Delta r, \cdot]
$$

where $\Delta r \in \Omega^{1}\left(W^{2}\left(E n d_{V}\right)\right)$. In other words any deviations of the connections $\partial^{s}$ and $\partial^{V}$ can be absorbed into the form $r \in \Omega^{1}\left(W^{2}\left(E n d_{V}\right)\right)$.

Proof. See page 151 in [9].

Let us consider the affine subspace $I_{N} \oplus \Gamma\left(W^{1}\left(E n d_{V}\right)\right)$ in $\Gamma\left(W\left(E n d_{V}\right)\right)$ consisting of the sums $U=I_{N}+U_{1}$, where $I_{N}$ is the identity endomorphism of $V$ and $U_{1}$ is an arbitrary element in $\Gamma\left(W^{1}\left(E n d_{V}\right)\right)$. It is straightforward that

Proposition 2. The affine subspace $I_{N} \oplus \Gamma\left(W^{1}\left(E n d_{V}\right)\right) \subset \Gamma\left(W\left(E n d_{V}\right)\right)$ is a subgroup in the group of invertible elements of $\Gamma\left(W\left(E n d_{V}\right)\right)$.

We are now ready to give the following

Definition 4. Two Fedosov connections

$$
D=\nabla+\frac{1}{\hbar}[A, \cdot], \quad \widetilde{D}=\nabla+\frac{1}{\hbar}[\widetilde{A}, \cdot]
$$

are called equivalent if there exists an element $U \in I_{N} \oplus \Gamma\left(W^{1}\left(E n d_{V}\right)\right)$ such that

$$
\widetilde{D}=D+\left[U^{-1} \circ D U, \cdot\right]
$$

or equivalently $\widetilde{A}=U^{-1} \circ A \circ U+\hbar U^{-1} \circ \nabla U$. 
Let us remark that the Fedosov connection (17) can be rewritten as

$$
D=\nabla-\delta+\frac{1}{\hbar}[r, \cdot]
$$

where

$$
\delta=\frac{1}{\hbar}\left[d x^{i} \omega_{i j}(x) y^{j}, \cdot\right]=d x^{i} \frac{\partial}{\partial y^{i}}
$$

is the Koszul derivation of the algebra $\Omega^{\bullet}\left(W\left(E n d_{V}\right)\right)$.

For our purposes we will need the homotopy operator for the Koszul differential $\delta$

$$
\delta^{-1} a=y^{k} i\left(\frac{\partial}{\partial x^{k}}\right) \int_{0}^{1} a(x, \hbar, t y, t d x) \frac{d t}{t},
$$

where $i\left(\partial / \partial x^{k}\right)$ denotes the contraction of an exterior form with the vector field $\partial / \partial x^{k}$, and $\delta^{-1}$ is extended to $\Gamma\left(W\left(E n d_{V}\right)\right)$ by zero.

Simple calculations show that $\delta^{-1}$ is indeed the homotopy operator for $\delta$, namely

$$
a=\sigma(a)+\delta \delta^{-1} a+\delta^{-1} \delta a, \quad \forall a \in \Omega\left(W\left(E n d_{V}\right)\right)
$$

where $\sigma$ is the natural projection

$$
\sigma(a)=\left.a\right|_{y=0, d x=0}, \quad a \in \Omega^{\bullet}\left(W\left(E n d_{V}\right)\right)
$$

from $\Omega^{\bullet}\left(W\left(E n d_{V}\right)\right)$ onto the algebra of endomorphisms $E n d_{V}((\hbar))$.

The proof of the following theorem is contained in section 5.3 of [9]. (More precisely, see theorem 5.3.3 and remarks at the end of section 5.3)

Theorem 2 (Fedosov, [9]). If $\partial^{s}$ is a symplectic connection on $\mathcal{M}, \partial^{V}$ is a connection on $V$ and $\Omega_{\hbar}$ is a series of closed two-forms in $\hbar \Omega^{2}(M)[[\hbar]]$ then

(1) One can construct a nilpotent derivation $D=\nabla+\hbar^{-1}\left[r-d x^{i} \omega_{i j}(x) y^{j}, \cdot\right]$ whose Fedosov-Weyl curvature (18) is equal to $C^{W}=-\omega+\Omega_{\hbar}$ and the element $r \in \Omega^{1}\left(W^{2}\left(E n d_{V}\right)\right)$ satisfies the normalization condition

(2) Given a Fedosov connection (17) one can construct a vector space isomorphism $\lambda$

$$
\lambda: \operatorname{End}_{V}((\hbar)) \stackrel{\longrightarrow}{\rightarrow} \Gamma_{D}\left(W\left(\operatorname{End}_{V}\right)\right)
$$

from $\operatorname{End}_{V}((\hbar))$ to the algebra $\Gamma_{D}\left(W\left(\operatorname{End}_{V}\right)\right)$ of flat sections of $W\left(E_{n} d_{V}\right)$ with respect to $D$. The product in $\operatorname{End}_{V}((\hbar))$ induced via the isomorphism $\lambda$ is the desired star-product (12)

(3) Two Fedosov connections $D$ and $\widetilde{D}$ whose Fedosov-Weyl curvatures represent the same De Rham cohomology class in $H_{D R}^{2}(\mathcal{M})[[\hbar]]$ are equivalent in the sense of definition 4. If the equivalence between $D$ and $\widetilde{D}$ is established by an element $U \in I_{N} \oplus \Gamma\left(W^{1}\left(E n d_{V}\right)\right)$ then the map

$$
a \mapsto U \circ a \circ U^{-1}
$$


gives an isomorphism from the algebra $\Gamma_{\widetilde{D}}\left(W\left(E n d_{V}\right)\right)$ of flat sections of $\widetilde{D}$ to the algebra $\Gamma_{D}\left(W\left(E^{2} d_{V}\right)\right)$ of flat sections of $D$.

Remark 1. The construction of the Fedosov connection is functorial in the sense that if $u$ is an isomorphism from $V$ to $\widetilde{V}$ then

$$
\widetilde{D}=D+\left[u D\left(u^{-1}\right), \cdot\right]
$$

is a Fedosov connection in $\Omega^{\bullet}\left(W\left(E n d_{\widetilde{V}}\right)\right)$. Moreover, the corresponding isomorphisms

$$
\lambda: \operatorname{End}_{V}((\hbar)) \stackrel{\Im}{\rightarrow} \Gamma_{D}\left(W\left(\operatorname{End}_{V}\right)\right), \quad \widetilde{\lambda}: \operatorname{End}_{\widetilde{V}}((\hbar)) \stackrel{\rightrightarrows}{\rightarrow} \Gamma_{\widetilde{D}}\left(W\left(\operatorname{End}_{\widetilde{V}}\right)\right)
$$

are related by the formula

$$
\widetilde{\lambda}(a)=u \lambda\left(u^{-1} a u\right) u^{-1}, \quad a \in \operatorname{End}_{\widetilde{V}} .
$$

Remark 2. If $V$ is a trivial vector bundle $L_{1}$ of $\operatorname{rank} N=1$ then the isomorphism $\lambda(28)$ gives a star-product $*$ in the algebra of functions $C(\mathcal{M})((\hbar))$. Due to the result of $\mathrm{P} . \mathrm{Xu}[19]$, any star-product in $C(\mathcal{M})((\hbar))$ is equivalent to the one obtained via Fedosov's procedure ${ }^{1}$. In particular, the cohomology class of the Fedosov-Weyl curvature (18) is a well-defined characteristic class of a star-product in $C(\mathcal{M})((\hbar))$. This characteristic class is referred to as the Deligne-Fedosov class.

Let us prove here an important technical lemma which might as well have an independent interest

Lemma 1. Let $V$ be a vector bundle over the symplectic manifold $\mathcal{M}$ and $D$ be a Fedosov connection (17) with the form $r=A+d x^{i} \omega_{i j}(x) y^{j}$ satisfying the normalization condition (27). If $q \in E^{2} d_{V}$ is an endomorphism of $V$ then

$$
\partial^{V} q=0 \quad \Rightarrow \quad D q=0 \text {. }
$$

In other words, if $q$ is $\partial^{V}$-flat then the isomorphism $\lambda$ (28) sends the $q$ to itself.

Proof. Since $q$ does not depend on $y^{\prime} s$ and $\partial^{V} q=0$ we have that $\nabla q=\delta q=$ 0 . Hence, $D q=\hbar^{-1}[r, q]$ and it suffices to show that the commutator $[r, q]$ is vanishing. Since $D$ is nilpotent $D[r, q]=0$.

The operator $\delta^{-1}(24)$ is not a derivation of algebra $\Omega^{\bullet}\left(W\left(E n d_{V}\right)\right)$. However, since $q$ does not depend on $y^{\prime} s$ and $\delta^{-1} r=0$,

$$
\delta^{-1}[r, q]=0 .
$$

Thus if $\eta=[r, q] \in \Omega^{1}\left(W\left(E n d_{V}\right)\right)$ we have

$$
\delta \eta=\nabla \eta+\frac{1}{\hbar}[r, \eta], \quad \delta^{-1} \eta=0 .
$$

Therefore, applying (25) to $\eta$ we get

$$
\eta=\delta^{-1}\left(\nabla \eta+\frac{1}{\hbar}[r, \eta]\right) .
$$

\footnotetext{
${ }^{1}$ See paper [8], in which this result was extended to any smooth affine algebraic symplectic variety.
} 
The latter equation has the unique vanishing solution since $\delta^{-1}$ raises the degree in $y$. Thus the lemma is proven.

2.4. The Feigin-Felder-Shoikhet trace density map. Let as above $V$ be a vector bundle of rank $N$ over the symplectic manifold $\mathcal{M}$ and $*$ be a star-product (12) in the algebra $\operatorname{End}_{V}((\hbar))$. Then,

Definition 5. A trace density map trd is a $\mathbb{C}((\hbar))$-linear map

$$
\text { trd : } \operatorname{End}_{V}((\hbar)) \mapsto H^{2 n}(\mathcal{M})((\hbar))
$$

vanishing on commutators

$$
\operatorname{trd}(a * b-b * a)=0, \quad a, b \in \operatorname{End}_{V}((\hbar)) .
$$

We will show that if the star-product $*$ in $\operatorname{End}_{V}((\hbar))$ is obtained via the Fedosov procedure [9] then the Feigin-Felder-Shoikhet cocycle (6) provides us with a natural trace density map.

Let as above $D$ be the Fedosov connection (17) and $\lambda$ be the isomorphism (28). Since $\Theta_{2 n}^{N}$ (6) is a cocycle of $\mathfrak{g}=\mathfrak{g l}_{N}(\mathcal{W})$ relative to $\mathfrak{h}=\mathfrak{g l}_{N} \oplus \mathfrak{s p}_{2 n}$, we have the following well-defined map

$$
\Psi_{D}: \operatorname{End}_{V}((\hbar)) \mapsto \Omega^{2 n}(\mathcal{M})((\hbar)), \quad \Psi_{D}(a)=\frac{1}{\hbar^{n}} \Theta_{2 n}^{N}(A, \ldots, A, \lambda(a)),
$$

where $A \in \Omega^{1}\left(W\left(E n d_{V}\right)\right)$ is the one-form entering the definition of the Fedosov connection $D(17)$.

We assemble the required properties of the map $\Psi_{D}$ in the following

Theorem 3 (Feigin-Felder-Shoikhet, [10]). With the above notations the following statements hold:

i) If $V_{1}$ and $V_{2}$ are two vector bundles over $\mathcal{M}$ and $D_{1}, D_{2}$ are Fedosov connections on $W\left(E_{n d_{V_{1}}}\right)$ and $W\left(E n d_{V_{2}}\right)$, respectively, then for any a $\in E_{n d_{V_{1}}}$

$$
\Psi_{D_{1} \oplus D_{2}}(a \oplus 0)=\Psi_{D_{1}}(a),
$$

where 0 stands for the trivial endomorphism of $V_{2}$.

ii) For any pair of endomorphisms $a, b \in \operatorname{End}_{V}((\hbar))$

$$
\Psi_{D}(a * b-b * a) \in d \Omega^{2 n-1}(\mathcal{M})((\hbar)),
$$

where $d$ is the De Rham differential.

iii) Let $D$ and $\widetilde{D}$ be two equivalent Fedosov connections and $U \in I_{N} \oplus \Gamma\left(W^{1}\left(\right.\right.$ End $\left.\left._{V}\right)\right)$ be the element establishing their equivalence in the sense of (21). Then for any $a \in \operatorname{End}_{V}((\hbar))$

$$
\Psi_{\widetilde{D}}\left(\sigma\left(U^{-1} \circ \lambda(a) \circ U\right)\right)-\Psi_{D}(a) \in d \Omega^{2 n-1}(\mathcal{M})((\hbar)),
$$

where $\sigma$ is the projection (26) and $\lambda$ is the isomorphism (28).

iv) For the identity endomorphism $I_{N} \in$ End $_{V}$

$$
\Psi_{D}\left(I_{N}\right)-\frac{1}{\hbar^{n}} Q_{n}(C(A, A), \ldots, C(A, A)) \in d \Omega^{2 n-1}(\mathcal{M})((\hbar)),
$$


where $Q_{n} \in\left(\left(S^{n} \mathfrak{h}\right)^{*}\right)^{\mathfrak{h}}$ is the $n$-th component of the adjoint invariant form (11), $A \in \Omega^{1}\left(W\left(E n d_{V}\right)\right)$ is the one-form entering the definition of the Fedosov connection $D$ (17) and $C$ is the fiberwise "curvature" (7) of the projection (9).

Proof. Statement $i$ ) is obvious from the construction of the cocycle $\Theta_{2 n}^{N}(1)$, (5), (6). Statements $i i), i i i)$, and $i v$ ) are proven in [10] for the case when the bundle $V$ is trivial. Statements $i i)$ and $i i i)$ follow from the fact $\Theta_{2 n}^{N}$ is a cocycle relative to $\mathfrak{h}=\mathfrak{g l}_{N} \oplus \mathfrak{s p}_{2 n}$ and statement $i v$ ) is a consequence of (10). These results of [10] are generalized to the non-trivial bundle $V$ in a straightforward manner since we always deal with the Lie algebra cochains relative to $\mathfrak{h}=\mathfrak{g l}_{N} \oplus$ $\mathfrak{s p}_{2 n}$.

As an immediate consequence of this theorem we get that

Corollary 1. If $*$ is the star-product in $\operatorname{End}_{V}((\hbar))$ obtained via the isomorphism $\lambda$ (28) then

$$
\operatorname{trd}(a)=\left[\Psi_{D}(a)\right]: \operatorname{End}_{V}((\hbar)) \mapsto H^{2 n}(\mathcal{M})((\hbar))
$$

is a trace density map.

In what follows we refer to (30) as the Feigin-Felder-Shoikhet (FFS) trace density map.

\section{The local version of the algebraic index theorem}

Let $\mathcal{M}$ be either a smooth real symplectic manifold of dimension $2 n$ or a smooth affine algebraic variety (over $\mathbb{C}$ ) of the complex dimension $2 n$ endowed with an algebraic symplectic form. Let $*$ be a star-product in the vector space $C(\mathcal{M})((\hbar))$ of smooth (resp. regular) functions on $\mathcal{M}$. Throughout this section we denote the algebra $(C(\mathcal{M})((\hbar)), *)$ by $\mathbb{A}$ and the subalgebra $(C(\mathcal{M})[[\hbar]], *)$ of $\mathbb{A}$ by $\mathbb{A}^{+}$

Due to remark 2 after theorem 2, we may safely assume that $*$ is obtained by Fedosov's procedure. Since in this case the vector bundle is trivial the Fedosov star-product depends only on the pair $\left(\partial^{s}, \Omega_{\hbar}\right)$, where $\partial^{s}$ is the symplectic connection on $\mathcal{M}$ and $\Omega_{\hbar} \in \hbar \Omega^{2}(\mathcal{M})[[\hbar]]$ is a series of closed two-forms. Let $D=\nabla+\hbar^{-1}[A, \cdot]$ be the corresponding Fedosov connection.

For any idempotent $P$ in the matrix algebra $\mathfrak{g l}_{N}\left(\mathbb{A}^{+}\right)$we assign the top degree De Rham cohomology class

$$
\operatorname{cl}(P)=\left[\Psi_{D}(P)\right] \in H^{2 n}(\mathcal{M})((\hbar)),
$$

where $D$ is naturally extended to the Fedosov connection on the Weyl algebra bundle $W\left(\operatorname{End}\left(L_{N}\right)\right)$ associated with the trivial bundle $L_{N}$ of rank $N$.

Due to statements $i$ ) and $i$ ) (31) gives a well-defined map

$$
c l: K_{0}\left(\mathbb{A}^{+}\right) \mapsto H^{2 n}(\mathcal{M})((\hbar))
$$

from the $K_{0}$-group of the algebra $\mathbb{A}^{+}$to $H^{2 n}(\mathcal{M})((\hbar))$.

Definition 6. The zeroth term $q=\left.P\right|_{\hbar=0}$ of an idempotent $P \in \mathfrak{g l}_{N}\left(\mathbb{A}^{+}\right)$is called the principal part of $P$. 
It is obvious that $q$ is an idempotent in the matrix algebra $\mathfrak{g l}_{N}(C(\mathcal{M}))$ and the operation of taking the principal part gives a well-defined principal symbol map

$$
\Xi: K_{0}\left(\mathbb{A}^{+}\right) \mapsto K_{0}(C(\mathcal{M})) .
$$

Due to the observation of L. Boutet de Monvel and V. Guillemin [4] for any idempotent $q$ in the matrix algebra $\mathfrak{g l}_{N}(C(\mathcal{M}))$ there exists an idempotent $P \in \mathfrak{g l}_{N}\left(\mathbb{A}^{+}\right)$whose principal part is $q$. (See the explicit formula for $P$ in [9], eq. $(6.1 .4))$

Using standard arguments of the index theory we can prove that the cohomology class $(31)$ of an idempotent $P \in \mathfrak{g l}_{N}\left(\mathbb{A}^{+}\right)$depends only on its principal part. For our purposes we need a slightly more general statement:

Proposition 3. Let $V$ be a vector bundle over $\mathcal{M}$ and $*$ be the Fedosov starproduct in End $_{V}[[\hbar]]$. Then if two idempotents $P_{1}, P_{2} \in\left(\right.$ End $\left._{V}[[\hbar]], *\right)$ have the same principal part

$$
\left.P_{1}\right|_{\hbar=0}=\left.P_{2}\right|_{\hbar=0}
$$

the cohomology classes $\left[\Psi_{D}\left(P_{1}\right)\right]$ and $\left[\Psi_{D}\left(P_{2}\right)\right]$ coincide.

Proof is a straightforward generalization of the arguments in the proof of theorem 6.1.3 in [9].

The precise dependence of the cohomology class $\operatorname{cl}(P)$ on the principal part $q$ of $P$ is given by the local algebraic index theorem

Theorem 4. For any element $\Pi \in K_{0}\left(\mathbb{A}^{+}\right)$the cohomology class cl $(\Pi)$ coincides with the top component of the cup product

$$
c l(\Pi)=\left[\hat{A}(\mathcal{M}) \exp \left(-\frac{F}{\hbar}\right) \operatorname{ch}(\Xi(\Pi))\right]_{2 n},
$$

of the $\hat{A}$-genus of $\mathcal{M}$, the exponent $e^{-\frac{F}{\hbar}}$ of the Deligne-Fedosov class

$$
F=\left[-\omega+\Omega_{\hbar}\right]
$$

of the star-product in $\mathbb{A}^{+}$and the Chern character ch $(\Xi(\Pi))$ of the principal symbol $\Xi(\Pi)$ of $\Pi$.

Proof. Let $P$ be an idempotent in the matrix algebra $\mathfrak{g l}_{N}\left(\mathbb{A}^{+}\right)$and $q \in$ $\mathfrak{g l}_{N}(C(\mathcal{M}))$ be the principal part of $P$. As an idempotent endomorphism of the trivial bundle $L_{N}$ or rank $N, q$ defines a subbundle $E=\operatorname{Im} q \subset L_{N}$.

It suffices to prove that the $2 n$-form $\Psi_{D}(P)$ has the same cohomology class as the $2 n$-th component of the form

$$
\operatorname{det}\left(\frac{R / 2}{\sinh (R / 2)}\right)^{1 / 2} \exp \left(\frac{\omega-\Omega_{\hbar}}{\hbar}\right) \operatorname{tr} \exp \left(R^{E}\right)
$$

where $R$ is the curvature form of the symplectic connection $\partial^{s}, R^{E}$ is the curvature form of the vector bundle $E$, and $t r$ stands for the ordinary matrix trace. 
We start with an observation that $q$ is a flat section of $\operatorname{End}\left(L_{N}\right)$ with respect to the connection

$$
\partial^{L_{N}}=d+[q(d q)-(d q) q, \cdot] .
$$

Let $D^{L_{N}}$ be the Fedosov connection (17) corresponding to the initial symplectic connection $\partial^{s}$ on $\mathcal{M}$, the initial series $\Omega_{h}$ of closed two-forms and the connection (33) on the trivial bundle $L_{N}$. By claim 3 of theorem 2 there exists an element $U \in I_{N} \oplus W^{1}\left(E n d_{L_{N}}\right)$ which establishes the equivalence between the connections $D$ and $D^{L_{N}}$ in the sense of definition 4 . Therefore, by statement iii) of theorem 3

$$
\Psi_{D}(P)-\Psi_{D^{L_{N}}}(\widetilde{P}) \in d \Omega^{2 n-1}(\mathcal{M})((\hbar)),
$$

where $\widetilde{P}=\sigma\left(U^{-1} \circ P \circ U\right)$ is an idempotent in the algebra $\left(\operatorname{End}_{L_{N}}, \tilde{*}\right)$, with the star-product $\tilde{*}$ corresponding to the Fedosov connection $D^{L_{N}}$.

Notice that, $\widetilde{P}$ and $P$ have the same principal part $\left.\widetilde{P}\right|_{\hbar=0}=\left.P\right|_{\hbar=0}=q$. Let us consider $q$ as an element in the algebra $\left(E n d_{L_{N}}, \tilde{*}\right)$. By claim 1 of theorem 2 we may assume that $D^{L_{N}}$ satisfies normalization condition (27). Therefore, by lemma 1 the element $q$ is flat with respect to $D^{L_{N}}$, and hence $q$ is an idempotent of the algebra $\left(E n d_{L_{N}}, \tilde{*}\right)$. By proposition 3 we may safely assume that $\widetilde{P}=q$.

We recall that $E=\operatorname{Im} q \subset L_{N}$ is the subbundle of $L_{N}$, corresponding to the idempotent $q$ and denote by $\widetilde{E}$ the subbundle $\widetilde{E} \subset L_{N}$ corresponding to the complementary idempotent $I_{N}-q \in E n d_{L_{N}}$. Thus, our trivial vector bundle $L_{N}$ is isomorphic to the direct $\operatorname{sum} E \oplus \widetilde{E}$. Let us fix an isomorphism $u$

$$
u: L \stackrel{\longrightarrow}{\longrightarrow} E \oplus \widetilde{E} \text {. }
$$

Since $q_{u}=u q u^{-1} \in E n d_{E \oplus \widetilde{E}}$ is the projector onto $E$ along $\widetilde{E}$

$$
\left.q_{u}\right|_{\Gamma(E)((\hbar))}=I_{m},\left.\quad q_{u}\right|_{\Gamma(\widetilde{E})((\hbar))}=0,
$$

where $m$ is the rank of $E$ and $I_{m}$ is the identity endomorphism of $E$.

Due to remark 1 after theorem 2

$$
D^{E \oplus \widetilde{E}}=D^{L_{N}}+\left[u D^{L_{N}}\left(u^{-1}\right), \cdot\right]
$$

is the Fedosov connection on the Weyl algebra bundle $W\left(E n d_{E \oplus \widetilde{E}}\right)$.

Furthermore, $q_{u}=u q u^{-1}$ is flat with respect to $D^{E \oplus \widetilde{E}}$, and hence the connection $D^{E \oplus \widetilde{E}}$ preserves the following subspaces of $\operatorname{End}_{E \oplus \widetilde{E}}((\hbar))$

$$
\begin{aligned}
& \left\{a \in \operatorname{End}_{E \oplus \widetilde{E}}((\hbar))|a|_{\Gamma(E)((\hbar))} \subset \Gamma(E)((\hbar)),\left.\quad a\right|_{\Gamma(\widetilde{E})((\hbar))}=0\right\}, \\
& \left\{a \in \operatorname{End}_{E \oplus \widetilde{E}}((\hbar))|a|_{\Gamma(\widetilde{E})((\hbar))} \subset \Gamma(\widetilde{E})((\hbar)),\left.\quad a\right|_{\Gamma(E)((\hbar))}=0\right\} .
\end{aligned}
$$

The latter implies that $D^{E \oplus \widetilde{E}}$ is a direct sum $D^{E} \oplus D^{\widetilde{E}}$ of the Fedosov connections

$$
D^{E}=\nabla^{E}+\frac{1}{\hbar}\left[A^{E}, \cdot\right], \quad D^{\widetilde{E}}=\nabla^{\widetilde{E}}+\frac{1}{\hbar}\left[A^{\widetilde{E}}, \cdot\right]
$$


on the bundles $W\left(E n d_{E}\right)$ and $W\left(E n d_{\widetilde{E}}\right)$, respectively. Thus, due to (34) and statement $i$ ) of theorem 3 we have

$$
\Psi_{D^{L_{N}}}(\widetilde{P})=\Psi_{D^{E}}\left(I_{m}\right),
$$

where $I_{m}$ is as above the identity endomorphism of the vector bundle $E$.

Using statement $i v$ ) of theorem 3 we get that

$$
\Psi_{D^{E}}\left(I_{m}\right)-\frac{1}{\hbar^{n}} Q_{n}\left(C\left(A^{E}, A^{E}\right), \ldots, C\left(A^{E}, A^{E}\right)\right) \in d \Omega^{2 n-1}(\mathcal{M})((\hbar)),
$$

where $Q_{n} \in\left(\left(S^{n} \mathfrak{h}\right)^{*}\right)^{\mathfrak{h}}$ is the $n$-th component of the adjoint invariant form (11), $\mathfrak{h}=\mathfrak{g l}_{N} \oplus \mathfrak{s p}_{2 n}, A^{E} \in \Omega^{1}\left(W\left(E n d_{E}\right)\right)$ is the one-form of the Fedosov connection $D^{E}$ (36), and $C$ is the fiberwise "curvature" (7) of the projection (9).

To compute $C\left(A^{E}, A^{E}\right)$ we mention that since the Fedosov connection $D^{E \oplus \widetilde{E}}$ is obtained from the $D^{L_{N}}$ via conjugation (35) the connection $D^{E \oplus \widetilde{E}}$ has the same Fedosov-Weyl curvature (18)

$$
\hbar\left(R+R^{E \oplus \widetilde{E}}\right)+2 \nabla^{E \oplus \widetilde{E}} A^{E \oplus \widetilde{E}}+\frac{1}{\hbar}\left[A^{E \oplus \widetilde{E}}, A^{E \oplus \widetilde{E}}\right]=-\omega+\Omega_{\hbar} .
$$

Here $A^{E \oplus \widetilde{E}}$ is the one-form of the Fedosov connection $D^{E \oplus \widetilde{E}}, \nabla^{E \oplus \widetilde{E}}$ is the operator (16) corresponding to the symplectic connection $\partial^{s}$ and the connection $\partial^{E \oplus \widetilde{E}}$ on $E \oplus \widetilde{E}$ obtained from $\partial^{L_{N}}$ via the isomorphism $u$. Finally, $R^{E \oplus \widetilde{E}}$ is the curvature form of the connection $\partial^{E \oplus \widetilde{E}}$.

On the other hand the Fedosov connection $D^{E \oplus \widetilde{E}}$ is a direct sum of the Fedosov connections $D^{E}$ and $D^{\widetilde{E}}$. Hence,

$$
\hbar\left(R+R^{E}\right)+2 \nabla^{E} A^{E}+\frac{1}{\hbar}\left[A^{E}, A^{E}\right]=-\omega+\Omega_{\hbar},
$$

where $R^{E}$ is the curvature form of the connection $\partial^{E}$ on $E$ and $\nabla^{E}$ is the operator (16) corresponding to the symplectic connection $\partial^{s}$ on $\mathcal{M}$ and the connection $\partial^{E}$.

Notice that, the projection $\operatorname{pr}\left(A^{E}\right)$ may be non-zero. However, using the trick of proposition 1 we can absorb the terms that contribute to $\operatorname{pr}\left(A^{E}\right)$ into the operator $\nabla^{E}$. In this way we change both ${ }^{2}$ the symplectic connection $\partial^{s}$ on $\mathcal{M}$ and the connection $\partial^{E}$ on $E$, and therefore we change curvature forms $R$ and $R^{E}$. It is not hard to show that after this rearrangement equation (38) still holds.

Since $\operatorname{pr}\left(A^{E}\right)$ is now vanishing $\operatorname{pr} \nabla\left(A^{E}\right)=\nabla \operatorname{pr}\left(A^{E}\right)=0$. Thus, applying $p r$ to both sides of $(38)$ we get

$$
C\left(A^{E}, A^{E}\right)=\hbar^{2} R+\hbar^{2} R_{E}-\hbar\left(-\omega+\Omega_{\hbar}\right) .
$$

Substituting (39) into equation (37) we complete the proof of the theorem.

\footnotetext{
${ }^{2}$ Notice that, both the new Christoffel form $\Gamma$ and the new connection form $\Gamma^{E}$ become formal power series in $\hbar$.
} 


\section{Concluding remarks.}

We would like to mention that our result (theorem 4) is derived from the purely algebraic fact (10) about the cohomology of Lie algebra of matrices over the Weyl algebra. In this respect our approach is very reminiscent of finding "local" proofs of the Riemann-Roch-Hirzebruch theorem [1], [3], [5], [6], and [12]. Thus, in [1] and [3] the Riemann-Roch theorem for families of Riemann curves is deduced from purely algebraic facts about the cohomology of the Lie algebra of vector fields. In paper [12] this idea is generalized to higher dimensions.

The Bressler-Nest-Tsygan theorem [6] or the Riemann-Roch theorem for deformation quantizations, which we already mentioned in the introduction, is also a local statement based on purely algebraic relations between Hochschild, cyclic, and Lie algebra (co)homology. It seems that this result is the most general Riemann-Roch type theorem which can be proven in the symplectic setting using the methods of [6].

A very similar statement to our result is proposed in recent paper [11] by B. Feigin, A. Losev, and B. Shoikhet. In this paper the authors consider the algebra $\operatorname{Diff}(\mathcal{E})$ of holomorphic differential operators acting on sections of a holomorphic vector bundle $\mathcal{E}$ over a compact complex manifold $X$ of the complex dimension $n$. They give a tractable notion of Hochschild homology $H H_{\bullet}$ of $\operatorname{Diff}(\mathcal{E})$ for which $H H_{\bullet}(\operatorname{Diff}(\mathcal{E}))=H^{2 n-\bullet}(X, \mathbb{C})$ and construct a map

$$
\Lambda: \operatorname{Diff}(\mathcal{E}) \mapsto H^{2 n}(X, \mathbb{C})
$$

which induces an isomorphism $H H_{0}(\operatorname{Diff}(\mathcal{E})) \cong H^{2 n}(X, \mathbb{C})$. Then, using the original Riemann-Roch-Hirzebruch theorem and assuming that the Euler characteristic of $\mathcal{E}$ is nonzero the authors of [11] deduce that for any $\mathcal{D} \in \operatorname{Diff}(\mathcal{E})$, the pairing of $\Lambda(\mathcal{D})$ with the fundamental class $[X]$ coincides with the super-trace $\operatorname{str}(\mathcal{D})$ of $\mathcal{D}$.

We would like mention that the technique used in [11] to define the map (40) is very similar to the procedure of constructing the Hochschild cocycle (5). This technique was originally proposed in paper [14] and we suspect that it has an independent interest.

Notice that, since our proof of theorem 4 is purely algebraic, it can be generalized in a straightforward manner to the setting of the symplectic Lie algebroids [17]. In this case the local algebraic index theorem relates the cohomology classes of the De Rham complex associated with the corresponding Lie algebroid.

\section{Acknowledgements}

This work was done while the first author was a student and the second author was a mentor in the Summer Program for Undergraduate Research. We both express sincere thanks to Hartley Rogers who organizes this remarkable program at M.I.T. We thank Richard Melrose and all participants of his Spring analysis seminar for their interest and stimulating discussions. The second author also thanks Alberto Cattaneo and Giovanni Felder for useful conversations. We are grateful to Teal Guidici for criticisms concerning our English. This work is 
partially supported by the NSF grant DMS-9988796, the Grant for Support of Scientific Schools NSh-1999.2003.2, the grant INTAS 00-561 and the grant CRDF RM1-2545-MO-03.

\section{References}

[1] E. Arbarello, C. de Concini, V. Kac, and C. Procesi, Moduli spaces of curves and representation theory, Commun. Math. Phys. 117 (1988) 1-36.

[2] M. Atiyah and I. Singer, The index of elliptic operators, I, III, Ann. of Math. 87, 2 (1968) 484-530, 546-609.

[3] A. Beilinson and V. Schechtman, Determinant bundle and Virasoro algebras, Commun. Math. Phys. 118 (1988) 651-701.

[4] L. Boutet de Monvel and V. Guillemin, The spectral theory of Toeplitz operators, Ann. Math. Stud., Vol. 99, Princeton University Press, 1981.

[5] P. Bressler, R. Nest, and B. Tsygan, Riemann-Roch theorems via deformation quantization, I, Adv. Math. 167, no. 1 (2002) 1-25.

[6] _ Riemann-Roch theorems via deformation quantization, II, Adv. Math. 167, no. 1 (2002) 26-73.

[7] A. Connes, M. Flato, and D. Sternheimer, Closed star-products and cyclic cohomology, Lett. Math. Phys. 24 (1992) 1-12.

[8] V.A. Dolgushev, Hochschild cohomology versus De Rham cohomology without formality theorems, math.QA/0405177.

[9] B. Fedosov, Deformation quantization and index theory, Akademie Verlag, Berlin, 1996.

[10] B. Feigin, G. Felder, and B. Shoikhet, Hochschild cohomology of the Weyl algebra and trace in deformation quantization, math.QA/0311303.

[11] B. Feigin, A. Losev, and B. Shoikhet, Riemann-Roch-Hirzebruch theorem and topological quantum mechanics, math.QA/0401400.

[12] B. Feigin and B. Tsygan, Riemann-Roch theorem and Lie algebra cohomology I, Proceeding of the Winter School on Geometry and Physics (Srni, 1988), Rend. Circ. Mat. Palermo (2) Suppl. No. 21, (1989), 15-52.

[13] M. Kontsevich, Deformation quantization of Poisson manifolds, Lett. Math. Phys. 66 (2003) 157-216; q-alg/9709040.

[14] V. Lysov, Anticommutativity equation in topological quantum mechanics, JETP Lett. 76 (2002) 724-727; hep-th/0212005.

[15] R. Melrose, Star products and local line bundles. Preprint 2004.

[16] R. Nest and B. Tsygan, Algebraic index theorem, Commun. Math. Phys. 172, no. 2 (1995) $223-262$.

[17] _ Deformations of symplectic Lie algebroids, deformations of holomorphic symplectic structures, and index theorems, Asian J. Math. 5, no. 4 (2001), 599-635; math.QA/9906020.

[18] _ Formal versus analytic index theorems, Internat. Math. Res. Notices, no. 11 (1996) 557-564.

[19] P. Xu, Fedosov *-products and quantum momentum maps, Commun. Math. Phys. 197, 1 (1998) 167-197.

Department of Mathematics, Mit, 77 Massachusetts Ave, Cambridge, MA 021394307

E-mail address: pnchen@mit.edu, vald@mit.edu 